Dear Author,

Please, note that changes made to the HTML content will be added to the article before publication, but are not reflected in this PDF.

Note also that this file should not be used for submitting corrections. 


\title{
Is digit ratio (2D:4D) a reliable pointer to speech laterality?
}

\author{
Q1 John M. Hudson*, Jessica C. Hodgson \\ School of Psychology, University of Lincoln, Lincoln, United Kingdom
}

\section{H I G H L I G H T S}

- First study to examine the effects of prenatal testosterone on blood flow during speech.

- Prenatal testosterone as indicated by digit ratio was not related to speech representation.

- There was no association between sex and speech representation.

- The results are inconsistent with androgen theories of cerebral lateralisation.

\section{A R T I C L E I N F O}

Article history:

Received 2 September 2015

Received in revised form

19 December 2015

Accepted 25 December 2015

Available online $\mathrm{xxx}$

\section{Keywords:}

Digit ratio

2D:4D

Language lateralisation

Functional Transcranial Doppler

Prenatal testosterone

Callosal theory

GBG theory

\begin{abstract}
A B S T R A C T
The relative length of the second and fourth digits (2D:4D ratio) is sexually dimorphic and a retrospective biomarker of prenatal hormonal exposure. Low ratios indicate higher prenatal testosterone (pT) and lower estrogen exposure, whereas the reverse pattern is associated with high ratios. Elevated levels of $\mathrm{pT}$ exposure have long been thought to modulate hemispheric specialisation; subsequently many studies use the 2D:4D ratio as a proxy index for $\mathrm{pT}$ to examine the effects of prenatal hormonal exposure on lateralised cognitive abilities. Here we used Transcranial Doppler ultrasonography and digit ratio to investigate whether pT has an influence on speech laterality. We tested 34 right and 14 left handed adults. Our results indicate that speech representation is unrelated to digit characteristics and therefore purportedly $\mathrm{pT}$. We discuss these findings in relation to androgen theories of lateralisation.
\end{abstract}

(c) 2015 Published by Elsevier B.V.

\section{Introduction}

The origin of speech lateralisation in the human brain remains elusive. Androgen accounts of cerebral lateralisation hypothesise that exposure to higher levels of prenatal testosterone (pT) produce neuronal alterations which lead to structural and functional hemispheric asymmetries [19]. There is debate nevertheless, over how these processes may modulate speech laterality, with differing albeit not mutually exclusive perspectives postulating contrasting predictions. According to Geschwind-Behan-Galaburda's (GBG) theory of cerebral lateralisation [9], elevated pT exposure slows the development and cytoarchitecture of the left hemisphere whilst facilitating the generation of compensatory neural mechanisms and functional status of the right hemisphere. Thus it is

\footnotetext{
* * Corresponding author at. School of Psychology, College of Social Science, Brayford Pool, Lincoln LN6 7TS, United Kingdom. Fax: + 441522886026.

E-mail address: jhudson@lincoln.ac.uk (J.M. Hudson).
}

postulated that pT reduces the verbal ability of the left hemisphere and increases the likelihood of right hemisphere or bilateral speech representation (for a critique see [6]. In contrast, Callosal theory [24] posits that elevated levels of pT enhance the potential for lateralisation. According to this view, laterality is modulated by the size of the corpus callosum. Low indices of lateralisation are associated with greater callosal integrity and increased functional interhemispheric connectivity. Higher pT exposure is hypothesised to trigger axonal pruning of the corpus callosum which reduces the level of coherence between the hemispheres and increases the potential for typical language dominance.

A widely accepted retrospective measure of $\mathrm{pT}$ is the ratio between the lengths of the second and fourth digits (2D:4D ratio). Lower 2D:4D ratios, or in particular, the difference between the ratios of the left and right hands (dR-L) are associated with higher levels of pT exposure and lower levels of prenatal estrogen (pE) exposure; the reverse pattern is associated with high ratios [17]. The link between 2D:4D and prenatal sex steroids has further been demonstrated experimentally. [25] demonstrated that 
63 chondrocyte proliferation in the fourth digit is sensitive to the balance between $\mathrm{pT}$ and $\mathrm{pE}$. The process was shown to be enhanced by relative increases in $\mathrm{pT}-\mathrm{pE}$, whereas a relative increase in $\mathrm{pE}-\mathrm{pT}$ reduced the process. Notably, the modulation of 2D:4D by sex steroids was shown only to occur prenatally and was not influenced by circulating sex hormone levels (see also, [22]. A growing body of research has subsequently used digit ratio as a putative biomarker to examine the lateralisation effects of pT on a range of tasks generally associated with the left (e.g., [4] or right hemispheres (e.g., [5]. These studies however tend to deploy indirect functional measures from which laterality is inferred on the basis of handedness and acknowledged brain-behaviour relations. A limitation of this approach is that handedness does not correlate perfectly to speech laterality. Although it is well known that for most humans the left-hemisphere is dominant for speech and handedness, some right-handers, and many ambilaterals and lefthanders have bilateral or right-hemisphere representation [14]. Moreover, handedness is multidimensional and there is no clear consensus on the precise components of handedness that may be related to speech representation. Here we sought to investigate the effects of pT exposure on speech representation by measuring asymmetries in brain activation during speech production directly. In this study, changes in cerebral blood flow velocity (BFV) were unequivocally assessed with a functional Transcranial Doppler ultrasonography (fTCD) system. Functional TCD has high temporal resolution and constitutes a reliable, valid and economical technique for the assessment of language lateralisation. Lateralisation indices (LIs) measured with fTCD correlate highly to those measured with functional MRI [21]. Support for Callosal theory will be evident if an inverse relationship between 2D:4D ratio and strength of typical lateralisation is observed. A positive relationship between these variables would be consistent with the GBG hypothesis.

\section{Method}

\subsection{Participants}

Forty eight adults participated in the study, 24 males ( mean $=21.96$ years, $S D=7.03$ ) and 24 females (mean $=23.13$ years, $\mathrm{SD}=8.68$ ). We aimed to test a reasonable group of participants with atypical speech representation, so recruited more left-handers $(n=14)$ than would be expected in a representative sample. No participants had a known neurological disorder nor were any taking medication known to affect the CNS or circulatory system. None had impairments in language or reading. All had normal or corrected to normal vision and gave informed consent prior to participating in the study. All participants were of Caucasian origin apart from one male of African descent. His digit characteristics however were not unusual (within 1 SD) and so his data was retained. Participants were recruited through internal communications at the University of Lincoln and were paid $£ 6.00$ for helping with the research. The investigation was approved by the ethics committee of the School of Psychology, University of Lincoln.

\subsection{Digit ratio}

The lengths of the second and fourth fingers of each hand were measured from the ventral proximal crease to the tip with vernier calipers. Right and left 2D:4D ratios were calculated for each hand and also averaged across the hands to derive the mean 2D:4D value. To obtain $\mathrm{dR}-\mathrm{L}$, the left 2D:4D ratio was subtracted from the right 2D:4D ratio.

\subsection{Handedness questionnaire}

We used the same questionnaire as that deployed by [8] In short, respondents indicated their preferred hand (right, left, either) for executing 14 unimanual and 7 bimanual tasks. The questionnaire contains the same primary and secondary activities as those described by [1] and additional activities from [18]. A handedness quotient (HQ) was calculated according to $[($ right - left $) /($ right + left $) \times 100]$. Positive values indicated right-handedness, negative values indicated left-handedness. In addition, we classified hand preference according to Annett's [2] criteria for small samples.

\subsection{Speech laterality}

Fluctuations in cerebral BFV were measured with fTCD (DWL Doppler-Box ${ }^{\mathrm{TM}} \mathrm{X}$ : manufacturer, DWL Compumedics Germany $\mathrm{GmbH}$ ). The system records alterations in BFV within the right and left middle cerebral arteries (MCAs) during language production, using two 2-MHz transducer probes attached to a headset and positioned slightly superior to each zygomatic arch.

The fTCD task involved a word generation paradigm (see [12]. For each trial, a letter appeared on a computer screen and the participant was given $15 \mathrm{~s}$ to silently generate as many words as possible that began with the letter. During the following $5 \mathrm{~s}$ period they were asked to report the words aloud. A $35 \mathrm{~s}$ period of relaxation (baseline) then followed before the start of the next trial. There were 23 trials in total.

A MATLAB (Mathworks Inc., Sherborn, MA, USA) software package called dopOSCCI [3], 2012 was used to compute a laterality index (LI) for each participant. ${ }^{1}$ The LI was derived from the maximum peak difference between the right and left side within a $2 \mathrm{~s}$ period of interest, when compared to a baseline rest period of $10 \mathrm{~s}$ The activation window was centralized to the time point at which the left-right deviation was greatest within the period of interest (POI). In the present paradigm the POI ranged from 3 to $13 \mathrm{~s}$ following presentation of the stimulus letter.

\section{Results}

Speech laterality was determined by the protocol outlined by [13]. According to this criterion laterality is considered to be clear in all cases in which the LI deviates by $>2$ SE from 0 . Left-hemisphere (typical) or right-hemisphere (atypical) speech dominance is indicated by positive or negative indices respectively. Cases with an $\mathrm{LI}<2$ SE from 0 are categorized as having bilateral speech representation. Using these criteria the following distribution for speech representation was found: typical $85 \%$ (mean $\mathrm{LI}=3.10, \mathrm{SD}=1.47$ ), atypical $15 \%$ (mean $\mathrm{LI}=-1.78, \mathrm{SD}=0.92$ ). The $\mathrm{LI}$ for all participants deviated by $>2$ SE from 0 and therefore none were considered to have bilateral speech representation.

The Kolmogorov-Smirnov test denoted that the 2D:4D ratios deviated from normality $[D(48)=0.13, p=0.04]$, subsequently

\footnotetext{
${ }^{1}$ DopOSCCI makes a number of computations in order to summarize the fTCD data and advance the validity of measuring hemispheric differences in BFV. First, the numbers of samples were reduced by downsampling the data from $\sim 100 \mathrm{~Hz}$ to $25 \mathrm{~Hz}$. Second, variations in cardiac cycle which may contaminate task-related signals were corrected using a cardiac cycle integration technique. Third, data contaminated by movement or 'drift' were removed prior to normalization. Normalized epochs were subsequently screened and excluded as measurement artifacts if activation values exceeded the acceptable range ( $\pm 40 \%$ mean BFV). Fourth, to contro for physiological process that can influence BFV (e.g., breathing rate, arousal, cardiac cycle), the mean activation of the baseline period was subtracted from each individual epoch. Deviations in left versus right activity were therefore baseline corrected and reflect relative changes in BFV.
} 
non-parametric analyses are reported for these values. To examine the reliability of digit measurement we correlated the lengths of corresponding digits in left and right hands. Both 2D $[r=0.938, p=0.001]$ and $4 \mathrm{D}[r=0.832, p=0.001]$ measures were highly correlated. The median $2 \mathrm{D}: 4 \mathrm{D}$ for the whole sample was 1.00 (range $=0.95-1.22$ ). The $2 \mathrm{D}: 4 \mathrm{D}$ ratio of the right hand $(\mathrm{Mdn}=1.01$, range $=0.94-1.47)$ was greater $[z=-2.841, p=0.004$, $r=-0.29$ ] than that of the left hand $(\mathrm{Mdn}=0.98$, range $=0.88-1.07)$. Mann-Whitney tests showed no significant differences between males and females for left 2D:4D [ $U=201, z=-1.794, p=0.073]$ or right 2D:4D [ $U=273, z=-0.309, p=0.757]$. But as expected [17], dRL was significantly lower (one-tailed) $[U=202, z=-1.773, p=0.038$, $r=-0.26]$ for males $(\mathrm{Mdn}=0.002$, range $=-0.11-0.11)$ than females $(\mathrm{Mdn}=0.031$, range $=-0.03-0.50)$.

Spearman's rho correlations were performed to examine the relationship between $\mathrm{LI}$ and digit ratio. The correlation between LI and measures of the left 2D:4D [ $r=-0.058, p=0.697]$, the right 2D:4D $[r=0.141, p=0.339]$ and $\mathrm{dR}-\mathrm{L}[r=0.123, p=0.407]$ were not significant (see Fig. 1); dR-L only explained $1.5 \%$ of the variance in strength of lateralisation. Further, there was no difference in LIs between those particpants $(n=17)$ with lower $(<1 ; \operatorname{Mdn}=2.20$, range $=-2.70-6.63)$ and those $(n=31)$ with higher $(\geq 1 ; \operatorname{Mdn}=2.74$, range $=-3.37-6.61)$ ratios $[U=232.5, z=-0.668$, $p=0.504]$; nor did LIs differ $[U=221, z=-1.382, p=0.167]$ between male $(\mathrm{Mdn}=2.34$, range $=-3.37-5.70)$ and female participants $(\mathrm{Mdn}=2.77$, range $=-1.65-6.61)$.

We measured hand preference as a continuous variable (HQ) and also according to [2] catergorsiation which produced the following distribution: consistent right-handers (47.9\%), inconsistent right-handers (22.9\%), consistent left-handers (14.6\%) and inconsistent left-handers (14.6\%). Regression analyses showed that catergorical indices did not predict left 2D:4D $[F(3,47)=1.736$, $p=0.173]$, right $2 \mathrm{D}: 4 \mathrm{D}[F(3,47)=0.190, p=0.903$ or $\mathrm{dR}-\mathrm{L}[F(3$, $47)=0.195, p=0.899$ ]. Continuous measures of handedness also failed to predict 2D:4D or dR-L (all F's $<1$ ).

To examine the potential relationship between speech representation and the different variables a series of multiple regression analyses were performed. Because of a high level of collinearity the contribution of the left and right 2D:4D were examined seperately. With the $\mathrm{LI}$ as the dependent variable and sex, HQ dR-L and right 2D:4D entered as independent variables. The regression model was not significant $[F(4,47)=1.029, p=0.403]$ nor were any beta values significant. This pattern prevailed across the hands and also when HQ was replaced by Annett's hand usage categories [ $F$ $(6,47)=0.898, p=0.505]$. The relationship between degree of hand preference (HQ) and the degree of speech lateralisation (LI) was also non significant $[r=0.187, p=0.203]$.

\section{Discussion}

Although indices of pT have previously been shown to be related to language lateralisation [15], the observed asymmetric changes in BFV within the MCAs that occur during speech were not found to be related to 2D:4D characteristics. At a group level, participants with a low digit ratio were indistinguishable from those with a higher digit ratio on the basis of speech representation.

We found male participants had a lower dR-L than females, which is consistent with the view that the measure is sexually dimorphic and positively associated to the androgen receptor gene [17]. However, similar to [14], we did not find sex differences in speech laterality. If digit ratio is a reliable surrogate for pT the lack of a sex effect in speech representation would appear to be a challenge for androgen accounts of cerebral lateralisation. Given pT is generally higher in males-Callosal theory predicts stronger
(A) Shows the relationship between mean 2D:4D and speech laterality

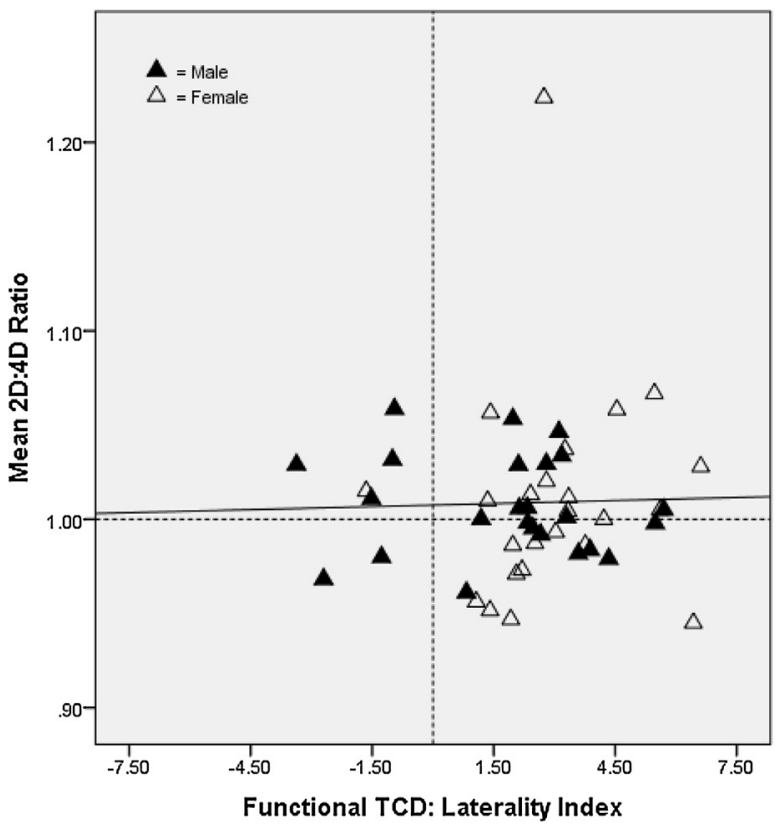

(B) Shows the relationship between dR-L and speech laterality

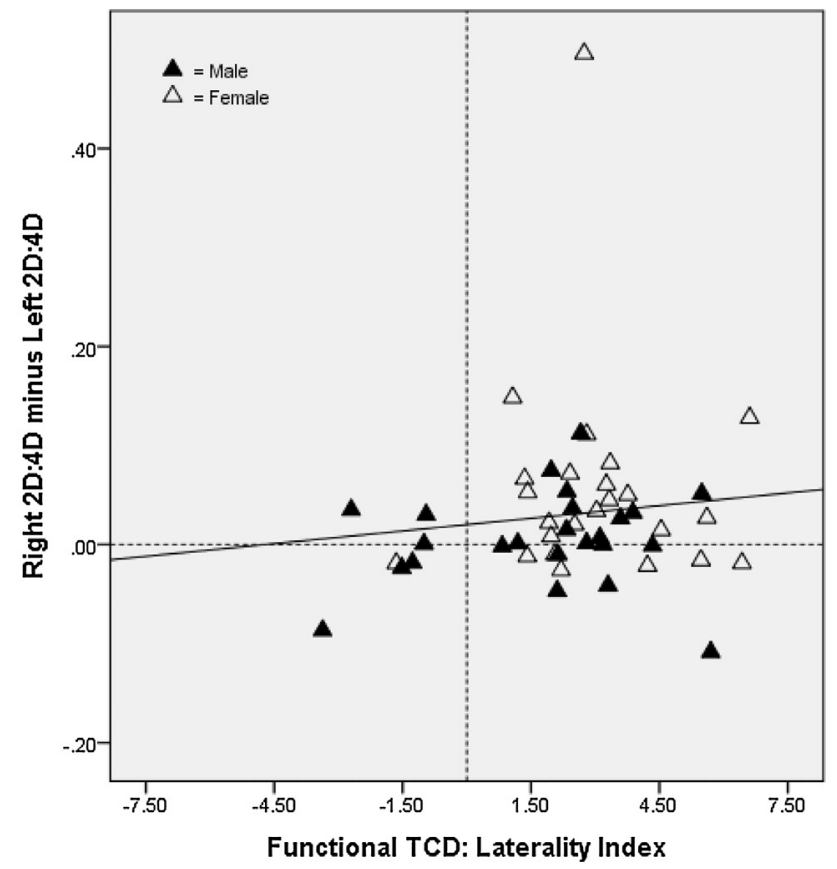

Fig. 1. The relationship between digit ratio and speech representation as a function of sex (A) Shows the relationship between mean 2D:4D and speech laterality. (B) Shows the relationship between $\mathrm{dR}-\mathrm{L}$ and speech laterality.

language lateralisation in males than females, whereas GBG theory predicts the reverse pattern.

Studies that examine the relationship between digit ratio and cognitive ability often assume laterality on the basis of hand preference. Preference measures however, have been criticised over reliability [16] and also validity [23]. The unambiguous measure of speech representation which we deployed is independent of hand preference. Indeed, $69 \%$ of our left handed participants, who would have been excluded from many research cohorts, were found to have typical left hemisphere speech representation. Similarly, three 
out of seven participants with atypical speech representation were classified as consistent right handers and therefore would normally be assumed to be left hemisphere dominant. Our results concur with a recent study by [20] who also observed that degree of hand preference does not predict degree of speech laterality.

To our knowledge, this is the first study to directly examine the relationship between 2D:4D characteristics and speech representation and the methods we deployed are widely considered to be robust. First, we took direct finger measurements which reduce the potential for distortion and averaged digit ratios across the hands [11] as well as analysing each hand separately [4]. Second, the word generation paradigm used here is the most widely adopted method for language lateralisation in fTCD investigations, and statements of laterality derived from this procedure are consistent with those observed with fMRI [7,21] and the Wada test [12]. Notwithstanding these strengths, it is possible that pT does modulate speech representation but digit ratio is not a reliable biomarker of prenatal hormonal exposure. It would therefore be worthwhile to examine the generality of the findings reported here. A potential approach would be to investigate the status of speech representation in individuals with genetic disorders which causes abnormal pT exposure (e.g., complete androgen insensitivity syndrome or congenital adrenal hyperplasia). Alternatively, a small number of developmental studies have measured pT exposure directly through the analysis of testosterone concentrations in amniotic fluid. Either of these approaches would have implications for further understanding the role of $\mathrm{pT}$ on speech representation.

\section{Uncited reference}

[10].

\section{Acknowledgments}

The research was supported by the Hessle Epilepsy Society. We thank Rachel Tremlin and Amy Holloway for assistance with data collection.

\section{References}

[1] M. Annett, A classification of hand preference by association analysis, Br. J. Psychol. 61 (3) (1970) 303-321.

[2] M. Annett, Handedness and brain asymmetry, in: The Right Shift Theory, Psychology Press, 2002.

[3] N.A. Badcock, G. Holt, A. Holden, D.V. Bishop, dopOSCCI: a functional transcranial Doppler ultrasonography summary suite for the assessment of cerebral lateralization of cognitive function, J. Neurosci. Methods 204 (2) (2012) 383-388.
[4] A.A. Beaton, S.V. Magowan, N.G. Rudling, Does handedness or digit ratio (2D:4D) predict lateralised cognitive ability? Pers. Individ. Diff. 52 (5) (2012) 627-631.

[5] V.J. Bourne, D.L. Gray, Hormone exposure and functional lateralisation: examining the contributions of prenatal and later life hormonal exposure, Psychoneuroendocrinology 34 (8) (2009) 1214-1221.

[6] M.P. Bryden, I.C. McManus, M.B. Bulman-Fleming, Evaluating the empirical support for the Geschwind-Behan-Galaburda model of cerebral lateralization, Brain Cogn. 26 (2) (1994) 103-167.

[7] M. Deppe, S. Knecht, K. Papke, H. Lohmann, H. Fleischer, W. Heindel, H. Henningsen, Assessment of hemispheric language lateralization: a comparison between fMRI and fTCD, J. Cereb. Blood Flow Metab. 2 (2000) 263-268.

[8] K.A. Flowers, J.M. Hudson, Motor laterality as an indicator of speech laterality, Neuropsychology 27 (2) (2013) 256-265.

[9] N. Geschwind, A. Galaburda, Cerebral lateralization: biological mechanisms, associations, and pathology: I -III. A hypothesis and a program for research, Arch. Neurol. 42 (1985) 428-459, 521-552, 634-654.

[10] J. Hönekopp, L. Bartholdt, L. Beier, A. Liebert, Second to fourth digit length ratio (2D:4D) and adult sex hormone levels: new data and a meta-analytic review, Psychoneuroendocrinology 32 (4) (2007) 313-321.

[11] C. Jackson, Prediction of hemispheric asymmetry as measured by handedness from digit length and 2D: 4D digit ratio, Laterality 13 (1) (2008) 34-50.

[12] S. Knecht, M. Deppe, A. Ebner, H. Henningsen, T. Huber, H. Jokeit, E.B. Ringelstein, Noninvasive determination of language lateralization by functional transcranial Doppler sonography: a comparison with the Wada test, Stroke 29 (1) (1998) 82-86

[13] S. Knecht, B. Dräger, A. Flöel, H. Lohmann, C. Breitenstein, M. Deppe, E.B Ringelstein, Behavioural relevance of atypical language lateralization in healthy subjects, Brain 8 (2001) 1657-1665.

[14] S. Knecht, B. Dräger, M. Deppe, L. Bobe, H. Lohmann, A. Floel, H. Henningsen, Handedness and hemispheric language dominance in healthy humans, Brain 123 (12) (2000) 2512-2518.

[15] J.M. Lust, R.H. Geuze, C. Van de Beek, P.T. Cohen-Kettenis, A. Bouma, T.G. Groothuis, Differential effects of prenatal testosterone on lateralization of handedness and language, Neuropsychology 25 (5) (2011) 581.

[16] E.R. McMeekan, W. Lishman, Retest reliabilities and interrelationship of the Annett hand preference questionnaire and the Edinburgh handedness inventory, Brit. J. Psychol. 66 (1) (1975) 53-59.

[17] J.T. Manning, P.E. Bundred, D.J. Newton, B.F. Flanagan, The second to fourth digit ratio and variation in the androgen receptor gene, Evol. Hum. Behav. 24 (6) (2003) 399-405

[18] R.C. Oldfield, Handedness in musicians, Br. J. Psychol. 60 (1) (1969) 91-99.

[19] C.L. Sisk, K.M. Schultz, J.L. Zehr, Puberty: a finishing school for male social behavior, Ann. N. Y. Acad. Sci. 1007 (1) (2003) 189-198.

[20] M. Somers, M.F. Aukes, R.A. Ophoff, M.P. Boks, W. Fleer, R.S. Kahn, I.E. Sommer, On the relationship between degree of hand-preference and degree of language lateralization, Brain Lang. 144 (2015) 10-15.

[21] M. Somers, S.F. Neggers, K.M. Diederen, M.P. Boks, R.S. Kahn, I.E. Sommer, The measurement of language lateralization with functional transcranial Doppler and functional MRI: a critical evaluation, Front. Hum. Neurosci. 5 (31) (2011).

[22] M. Voracek, Special issue preamble: digit ratio (2D:4D) and individual differences research, Pers. Individ. Dif. 51 (4) (2011) 367-370.

[23] S.M. Williams, Handedness inventories: Edinburgh versus Annett, Neuropsychology 5 (1) (1991) 43.

[24] S.F. Witelson, R.S. Nowakowski, Left out axons make men right: a hypothesis for the origin of handedness and functional asymmetry, Neuropsychologia 29 (4) (1991) 327-333.

[25] Z. Zheng, M.J. Cohn, Developmental basis of sexually dimorphic digit ratios, Proc. Natl. Acad. Sci. 108 (39) (2011) 16289-16294. 